Kragujevac Journal of Mathematics

Volume 42(4) (2018), Pages 591-605.

\title{
LOWER EXTREMITIES FOR GENERALIZED NORMALIZED $\delta$-CASORATI CURVATURES OF BI-SLANT SUBMANIFOLDS IN GENERALIZED COMPLEX SPACE FORMS
}

\author{
MOHD. AQUIB ${ }^{1}$, MOHAMMAD HASAN SHAHID $^{1}$, AND MOHAMMED JAMALI ${ }^{2}$
}

\begin{abstract}
In this paper, we obtain the inequalities for the generalized normalized $\delta$-Casorati curvature and the normalized scalar curvature for different submanifolds in generalized complex space form, which is based on an optimization procedure involving a quadratic polynomial in the components of the second fundamental form and characterizes the submanifolds on which equalities hold. We also develop, the same inequalities for semi-slant, hemi-slant, CR, slant, invariant and anti-invariant submanifolds in the same target space and consider the equality case. Moreover, we obtain a geometric inequality involving Casorati curvature for warped product bi-slant submanifolds in same ambient and obtain an obstruction result.
\end{abstract}

\section{INTRODUCTION}

The theory of Chen invariants, which establish the simple relationships between the main intrinsic invariants and the main extrinsic invariants of the submanifolds is one of the most interesting research areas of differential geometry started by Chen [5] in 1993. In the initial papers, Chen established inequalities between the scalar curvature, the sectional curvature(intrinsic invariants) and the squared norm of the mean curvature(the main extrinsic invariant) of a submanifold in a real space form. The same author obtained the inequalities for submanifolds between the $k$-Ricci curvature, the squared mean curvature, and the shape operator in the real space form with arbitrary codimension [4]. Since then, different geometers proved the similar inequalities for different submanifolds and ambient spaces $[13,15]$.

\footnotetext{
Key words and phrases. Casorati curvature, generalized complex space form, scalar curvature, warped product.

2010 Mathematics Subject Classification. Primary: 53B05. Secondary: 53B20, 53C40.

Received: May 08, 2017.

Accepted: July 26, 2017.
} 
The Casorati curvature(extrinsic invariant) of a submanifold of a Riemannian manifold, introduced by Casorati is defined as the normalized square length of the second fundamental form [3]. The concept of Casorati curvature extends the concept of the principal direction of a hypersurface of a Riemannian manifold [9]. The geometrical meaning and the importance of the Casorati curvature has been discussed by distinguished geometers $[6,12,19]$. Therefore, it attracts the attention of geometers to obtain the optimal inequalities for the Casorati curvatures of the submanifolds of different ambient spaces $[1,7,14]$.

In this article, we study the inequalities which relates the generalized normalized $\delta$-Casorati curvature and the normalized scalar curvature for different submanifolds in generalized complex space form and consider the equality case of the inequality. We also obtain, a geometric inequality involving Casorati curvature for warped product submanifolds in same ambient and obtain a non-existence result.

\section{Preliminaries}

Let $\bar{M}$ be an almost Hermitian manifold with an almost complex structure $J$ and a Riemannian metric $g$. An almost Hermitian manifold is said to be a nearly Kaehler manifold if $\left(\bar{\nabla}_{X} J\right) X=0$ and becomes a Kaehler manifold if $\bar{\nabla} J=0$ for all $X \in T \bar{M}$, where $\bar{\nabla}$ is the Levi-Civita connection of the Riemannian metric $g$.

Tricerri and Vanhecke [16] introduced the concept of generalized complex space form as a generalization of the complex space form. An almost Hermitian manifold $\bar{M}$ is called the generalized complex space form, denoted by $\bar{M}\left(f_{1}, f_{2}\right)$, if the Riemannian curvature tensor $\bar{R}$ satisfies

$$
\begin{aligned}
\bar{R}(X, Y) Z= & f_{1}\{g(Y, Z) X-g(X, Z) Y\}+f_{2}\{g(X, J Z) J Y \\
& -g(Y, J Z) J X+2 g(X, J Y) J Z\},
\end{aligned}
$$

for all $X, Y, Z \in T \bar{M}$, where $f_{1}$ and $f_{2}$ are smooth functions on $\bar{M}\left(f_{1}, f_{2}\right)$.

Let $M$ be an $n$-dimensional submanifold of a generalized complex space form $\bar{M}\left(f_{1}, f_{2}\right)$ of complex dimension $m$. Let $\nabla$ and $\bar{\nabla}$ be the Levi-Civita connection on $M$ and $\bar{M}\left(f_{1}, f_{2}\right)$ respectively. The Gauss and Weingarten equations are defined as

$$
\begin{gathered}
\bar{\nabla}_{X} Y=\nabla_{X} Y+h(X, Y), \\
\bar{\nabla}_{X} \xi=-S_{\xi} X+\nabla_{X}^{\perp} Y,
\end{gathered}
$$

for vector fields $X, Y \in T M$ and $\xi \in T^{\perp} M$, where $h, S$ and $\nabla^{\perp}$ are the second fundamental form, the shape operator and the normal connection respectively. The second fundamental form and the shape operator are related by the following equation

$$
g(h(X, Y), \xi)=g\left(S_{\xi} X, Y\right),
$$

for vector fields $X, Y \in T M$ and $\xi \in T^{\perp} M$.

The equation of Gauss is given by

(2.2) $\bar{R}(X, Y, Z, W)=R(X, Y, Z, W)+g(h(X, Z), h(Y, W))-g(h(X, W), h(Y, Z))$, 
TABLE 1. Definition

\begin{tabular}{|l|l|l|l|l|l|l|}
\hline S.N. & $\bar{M}$ & $M$ & $D_{1}$ & $D_{2}$ & $\theta_{1}$ & $\theta_{2}$ \\
\hline$(1)$ & $\bar{M}$ & bi-slant & slant & slant & slant angle & slant angle \\
\hline$(2)$ & $\bar{M}$ & semi-slant & invariant & slant & 0 & slant angle \\
\hline$(3)$ & $\bar{M}$ & hemi-slant & slant & anti-invariant & slant angle & $\frac{\pi}{2}$ \\
\hline$(4)$ & $\bar{M}$ & CR & invariant & anti-invariant & 0 & $\frac{\pi}{2}$ \\
\hline$(5)$ & $\bar{M}$ & slant & either $D_{1}=0$ or $D_{2}=0$ & either $\theta_{1}=\theta_{2}=\theta$ or $\theta_{1}=\theta_{2} \neq \theta$ \\
\hline
\end{tabular}

for $X, Y, Z, W \in T M$, where $\bar{R}$ and $R$ represent the curvature tensor of $\bar{M}\left(f_{1}, f_{2}\right)$ and $M$ respectively.

Let $M$ be an $n$-dimensional submanifold of a generalized complex space form $\bar{M}\left(f_{1}, f_{2}\right)$ of complex dimension $m$. For any tangent vector field $X \in T M$, we can write $J X=P X+Q X$, where $P$ and $Q$ are the tangential and normal components of $J X$ respectively. If $P=0$, the submanifold is said to be an anti-invariant submanifold and if $Q=0$, the submanifold is said to be an invariant submanifold. The squared norm of $P$ at $p \in M$ is defined as

$$
\|P\|^{2}=\sum_{i, j=1}^{n} g^{2}\left(J e_{i}, e_{j}\right),
$$

where $\left\{e_{1}, \ldots, e_{n}\right\}$ is any orthonormal basis of the tangent space $T_{p} M$.

A submanifold $M$ of an almost Hermitian manifold $\bar{M}$ is said to be a slant submanifold if for any $p \in M$ and a non zero vector $X \in T_{p} M$, the angle between $J X$ and $T_{p} M$ is constant, i.e., the angle does not depend on the choice of $p \in M$ and $X \in T_{p} M$. The angle $\theta \in\left[0, \frac{\pi}{2}\right]$ is called the slant angle of $M$ in $\bar{M}$.

A submanifold $M$ of an almost Hermitian manifold $\bar{M}$ is said to be a bi-slant submanifold, if there exist two orthogonal distributions $D_{1}$ and $D_{2}$, such that

(i) $T M$ admits the orthogonal direct decomposition, i.e., $T M=D_{1}+D_{2}$;

(ii) for $i=1,2, D_{i}$ is the slant distribution with slant angle $\theta_{i}$.

In fact, semi-slant submanifolds, hemi-slant submanifolds, CR-submanifolds, slant submanifolds can be obtained from bi-slant submanifolds in particular. We can see the case in the following table: Invariant and anti-invariant submanifolds are the slant submanifolds with slant angle $\theta=0$ and $\theta=\frac{\pi}{2}$ respectively. If $0<\theta<\frac{\pi}{2}$, then slant submanifold is called proper slant submanifold. It is said to be proper bi-slant if $\theta_{i}$ lies between 0 and $\frac{\pi}{2}$.

If $M$ is a bi-slant submanifold in a generalized complex space form $\bar{M}\left(f_{1}, f_{2}\right)$, then one easily can see that

$$
\|P\|^{2}=\sum_{i, j=1}^{n} g^{2}\left(P e_{i}, e_{j}\right)=2\left(d_{1} \cos ^{2} \theta_{1}+d_{2} \cos ^{2} \theta_{2}\right),
$$

where $\operatorname{dim} D_{1}=2 d_{1}$ and $\operatorname{dim} D_{2}=2 d_{2}$. 
Let $M$ be a Riemannian manifold and $K(\pi)$ denotes the sectional curvature of $M$ of the plane section $\pi \subset T_{p} M$ at a point $p \in M$. If $\left\{e_{1}, \ldots, e_{n}\right\}$ and $\left\{e_{n+1}, \ldots, e_{2 m}\right\}$ be the orthonormal basis of $T_{p} M$ and $T_{p}^{\perp} M$ at any $p \in M$, then the scalar curvature $\tau$ at that point is given by

$$
\tau(p)=\sum_{1 \leq i<j \leq n} K\left(e_{i} \wedge e_{j}\right)
$$

and the normalized scalar curvature $\rho$ is defined as

$$
\rho=\frac{2 \tau}{n(n-1)} \text {. }
$$

The mean curvature vector denoted by $H$ is defined as

$$
H=\frac{1}{n} \sum_{i=1}^{n} h\left(e_{i}, e_{i}\right) \text {. }
$$

We also put

$$
h_{i j}^{\gamma}=g\left(h\left(e_{i}, e_{j}\right), e_{\gamma}\right), \quad i, j \in 1,2, \ldots, n, \gamma \in\{n+1, n+2, \ldots, 2 m\} .
$$

The squared norm of the mean curvature of the submanifold is defined by

$$
\|H\|^{2}=\frac{1}{n^{2}} \sum_{\gamma=n+1}^{2 m}\left(\sum_{i=1}^{n} h_{i i}^{\gamma}\right)^{2},
$$

and the squared norm of second fundamental form $h$ is denoted by $\mathcal{C}$ defined as

$$
\mathcal{C}=\frac{1}{n} \sum_{\gamma=n+1}^{2 m} \sum_{i, j=1}^{n}\left(h_{i j}^{\gamma}\right)^{2}
$$

known as Casorati curvature of the submanifold.

If we suppose that $L$ is an $r$-dimensional subspace of $T M, r \geq 2$, and $\left\{e_{1}, e_{2}, \ldots, e_{r}\right\}$ is an orthonormal basis of $L$, then the scalar curvature of the $r$-plane section $L$ is given as

$$
\tau(L)=\sum_{1 \leq \gamma<\beta \leq r} K\left(e_{\gamma} \wedge e_{\beta}\right)
$$

and the Casorati curvature $\mathcal{C}$ of the subspace $L$ is as follows

$$
\mathcal{C}(L)=\frac{1}{r} \sum_{\gamma=n+1}^{2 m} \sum_{i, j=1}^{r}\left(h_{i j}^{\gamma}\right)^{2} .
$$

A point $p \in M$ is said to be an invariantly quasi-umbilical point if there exist $2 m-n$ mutually orthogonal unit normal vectors $\xi_{n+1}, \ldots, \xi_{2 m}$ such that the shape operators with respect to all directions $\xi_{\gamma}$ have an eigenvalue of multiplicity $n-1$ and that for each $\xi_{\gamma}$ the distinguished eigen direction is the same. The submanifold is said to be an invariantly quasi-umbilical submanifold if each of its points is an invariantly quasi-umbilical point. 
The normalized $\delta$-Casorati curvature $\delta_{c}(n-1)$ and $\widehat{\delta}_{c}(n-1)$ are defined as

$$
\left[\delta_{c}(n-1)\right]_{p}=\frac{1}{2} \varrho_{p}+\frac{n+1}{2 n} \inf \left\{\mathcal{C}(L) \mid L: \text { a hyperplane of } T_{p} M\right\}
$$

and

$$
\left[\widehat{\delta}_{c}(n-1)\right]_{p}=2 \mathcal{C}_{p}+\frac{2 n-1}{2 n} \sup \left\{\mathfrak{C}(L) \mid L: \text { a hyperplane of } T_{p} M\right\} .
$$

For a positive real number $t \neq n(n-1)$, put

$$
a(t)=\frac{1}{n t}(n-1)(n+t)\left(n^{2}-n-t\right)
$$

then the generalized normalized $\delta$-Casorati curvatures $\delta_{c}(t ; n-1)$ and $\widehat{\delta}_{c}(t ; n-1)$ are given as

$$
\left[\delta_{c}(t ; n-1)\right]_{p}=t \mathcal{C}_{p}+a(t) \inf \left\{\mathcal{C}(L) \mid L: \text { a hyperplane of } T_{p} M\right\},
$$

if $0<t<n^{2}-n$, and

$$
\left[\widehat{\delta}_{c}(t ; n-1)\right]_{p}=r \mathfrak{C}_{p}+a(t) \sup \left\{\mathfrak{C}(L) \mid L: \text { a hyperplane of } T_{p} M\right\},
$$

if $t>n^{2}-n$.

\section{Generalized Normalized $\delta$-Casorati Curvature}

In this section we obtain the generalized normalized $\delta$-Casorati curvatures for different submanifolds in generalized complex space form.

Theorem 3.1. Let $M$ be a bi-slant submanifold of a generalized complex space form $\bar{M}\left(f_{1}, f_{2}\right)$. Then

(i) the generalized normalized $\delta$-Casorati curvature $\delta_{c}(t ; n-1)$ satisfies

$$
\rho \leq \frac{\delta_{c}(t ; n-1)}{n(n-1)}+f_{1}+\frac{6 f_{2}}{n(n-1)}\left(d_{1} \cos ^{2} \theta_{1}+d_{2} \cos ^{2} \theta_{2}\right),
$$

for any real number $t$ such that $0<t<n(n-1)$, and

(ii) the generalized normalized $\delta$-Casorati curvature $\widehat{\delta}_{c}(t ; n-1)$ satisfies

$$
\rho \leq \frac{\widehat{\delta}_{c}(t ; n-1)}{n(n-1)}+f_{1}+\frac{6 f_{2}}{n(n-1)}\left(d_{1} \cos ^{2} \theta_{1}+d_{2} \cos ^{2} \theta_{2}\right),
$$

for any real number $t>n(n-1)$. Moreover, the equality holds in (3.1) and (3.2) if and only if $M$ is an invariantly quasi-umbilical submanifold with trivial normal connection in $\bar{M}\left(f_{1}, f_{2}\right)$, such that with respect to suitable tangent orthonormal frame $\left\{e_{1}, \ldots, e_{n}\right\}$ and normal orthonormal frame $\left\{e_{n+1}, \ldots, e_{2 m}\right\}$, the shape operator $S_{r} \equiv$ 
$S_{e_{r}}, r \in\{n+1, \ldots, 2 m\}$, takes the following form

$$
S_{n+1}=\left(\begin{array}{cccccc}
a & 0 & 0 & \ldots & 0 & 0 \\
0 & a & 0 & \ldots & 0 & 0 \\
0 & 0 & a & \ldots & 0 & 0 \\
\vdots & \vdots & \vdots & \ddots & \vdots & \vdots \\
0 & 0 & 0 & \ldots & a & 0 \\
0 & 0 & 0 & \ldots & 0 & \frac{n(n-1)}{t} a
\end{array}\right), S_{n+2}=\cdots=S_{2 m}=0
$$

Proof. Let $\left\{e_{1}, \ldots, e_{n}\right\}$ and $\left\{e_{n+1}, \ldots, e_{2 m}\right\}$ be the orthonormal basis of $T_{p} M$ and $T_{p}^{\perp} M$ respectively at any point $p \in M$. Putting $X=W=e_{i}, Y=Z=e_{j}, i \neq j$ from (2.1), we have

$$
\begin{aligned}
\bar{R}\left(e_{i}, e_{j}, e_{j}, e_{i}\right)= & f_{1}\left\{g\left(e_{j}, e_{j}\right) g\left(e_{i}, e_{i}\right)-g\left(e_{i}, e_{j}\right) g\left(e_{j}, e_{i}\right)\right\} \\
& +f_{2}\left\{g\left(e_{i}, J e_{j}\right) g\left(J e_{j}, e_{i}\right)-g\left(e_{j}, J e_{j}\right) g\left(J e_{i}, e_{i}\right)\right. \\
& \left.+2 g\left(e_{i}, J e_{j}\right) g\left(J e_{j}, e_{i}\right)\right\} .
\end{aligned}
$$

From Gauss equation and (3.4), we have

$$
\begin{aligned}
R\left(e_{i}, e_{j}, e_{j}, e_{i}\right)= & f_{1}\left\{g\left(e_{j}, e_{j}\right) g\left(e_{i}, e_{i}\right)-g\left(e_{i}, e_{j}\right) g\left(e_{j}, e_{i}\right)\right\} \\
& +3 f_{2}\left\{g\left(e_{i}, J e_{j}\right) g\left(J e_{j}, e_{i}\right)\right\}-g\left(h\left(e_{i}, e_{j}\right), h\left(e_{j}, e_{i}\right)\right) \\
& +g\left(h\left(e_{i}, e_{i}\right), h\left(e_{j}, e_{j}\right)\right) .
\end{aligned}
$$

By taking summation $1 \leq i, j \leq n$ and using (2.3) and (3.5), we get

$$
2 \tau=n^{2}\|H\|^{2}-n \mathcal{C}+n(n-1) f_{1}+6 f_{2}\left(d_{1} \cos ^{2} \theta_{1}+d_{2} \cos ^{2} \theta_{2}\right) .
$$

Define the following function, denoted by $Q$, a quadratic polynomial in the components of the second fundamental form

$$
\mathcal{Q}=n(n-1) f_{1}+6 f_{2}\left(d_{1} \cos ^{2} \theta_{1}+d_{2} \cos ^{2} \theta_{2}\right)+t \mathcal{C}+a(t) \mathcal{C}(L)-2 \tau,
$$

where $L$ is the hyperplane of $T_{p} M$. Without loss of generality, we suppose that $L$ is spanned by $e_{1}, \ldots, e_{n-1}$, it follows from (3.6) and (3.7) that

$$
\mathcal{Q}=\frac{n+t}{n} \sum_{\gamma=n+1}^{2 m} \sum_{i, j=1}^{n}\left(h_{i j}^{\gamma}\right)^{2}+\frac{a(t)}{n-1} \sum_{\gamma=n+1}^{2 m} \sum_{i, j=1}^{n-1}\left(h_{i j}^{\gamma}\right)^{2}-\sum_{\gamma=n+1}^{2 m}\left(\sum_{i=1}^{n} h_{i i}^{\gamma}\right)^{2},
$$

which can be easily written as

$$
\begin{aligned}
\mathcal{Q}= & \sum_{\gamma=n+1}^{2 m} \sum_{i=1}^{n-1}\left[\left(\frac{n+t}{n}+\frac{a(t)}{n-1}\right)\left(h_{i i}^{\gamma}\right)^{2}+\frac{2(n+t)}{n}\left(h_{i n}^{\gamma}\right)^{2}\right] \\
& +\sum_{n+1}^{2 m}\left[2\left(\frac{n+t}{n}+\frac{a(t)}{n-1}\right) \sum_{(i<j)=1}^{n}\left(h_{i j}^{\gamma}\right)^{2}-2 \sum_{(i<j)=1}^{n} h_{i i}^{\gamma} h_{j j}^{\gamma}+\frac{t}{n}\left(h_{n n}^{\gamma}\right)^{2}\right] .
\end{aligned}
$$

From (3.8), we can see that the critical points

$$
h^{c}=\left(h_{11}^{n+1}, h_{12}^{n+1}, \ldots, h_{n n}^{n+1}, \ldots, h_{11}^{2 m}, \ldots, h_{n n}^{2 m}\right)
$$


of $\mathcal{Q}$ are the solutions of the following system of homogenous equations:

$$
\left\{\begin{array}{l}
\frac{\partial Q}{\partial h_{i i}^{\gamma}}=2\left(\frac{n+t}{n}+\frac{a(t)}{n-1}\right)\left(h_{i i}^{\gamma}\right)-2 \sum_{k=1}^{n} h_{k k}^{\gamma}=0, \\
\frac{\partial Q}{\partial h_{n n}^{\gamma}}=\frac{2 t}{n} h_{n n}^{\gamma}-2 \sum_{k=1}^{n-1} h_{k k}^{\gamma}=0, \\
\frac{\partial Q}{\partial h_{i j}^{\gamma}}=4\left(\frac{n+t}{n}+\frac{a(t)}{n-1}\right)\left(h_{i j}^{\gamma}\right)=0, \\
\frac{\partial Q}{\partial h_{i n}^{\gamma}}=4\left(\frac{n+t}{n}\right)\left(h_{i n}^{\gamma}\right)=0,
\end{array}\right.
$$

where $i, j=\{1,2, \ldots, n-1\}, i \neq j$, and $\gamma \in\{n+1, n+2, \ldots, 2 m\}$.

Hence, every solution $h^{c}$ has $h_{i j}^{\gamma}=0$ for $i \neq j$ and the corresponding determinant to the first two equations of the above system is zero. Moreover, the Hessian matrix of $\mathcal{Q}$ is of the following form

$$
\mathcal{H}(\mathcal{Q})=\left(\begin{array}{ccc}
H_{1} & O & O \\
O & H_{2} & O \\
O & O & H_{3}
\end{array}\right),
$$

where

$$
H_{1}=\left(\begin{array}{ccccc}
2\left(\frac{n+t}{n}+\frac{a(t)}{n-1}\right)-2 & -2 & \ldots & -2 & -2 \\
-2 & 2\left(\frac{n+t}{n}+\frac{a(t)}{n-1}\right)-2 & \ldots & -2 & -2 \\
\vdots & \vdots & \ddots & \vdots & \vdots \\
-2 & -2 & \ldots & 2\left(\frac{n+t}{n}+\frac{a(t)}{n-1}\right)-2 & -2 \\
-2 & -2 & \ldots & -2 & \frac{2 t}{n}
\end{array}\right),
$$

$H_{2}$ and $H_{3}$ are the diagonal matrices and $O$ is the null matrix of the respective dimensions. $\mathrm{H}_{2}$ and $\mathrm{H}_{3}$ are respectively given as

$$
H_{2}=\operatorname{diag}\left(4\left(\frac{n+t}{n}+\frac{a(t)}{n-1}\right), 4\left(\frac{n+t}{n}+\frac{a(t)}{n-1}\right), \ldots, 4\left(\frac{n+t}{n}+\frac{a(t)}{n-1}\right)\right),
$$

and

$$
H_{3}=\operatorname{diag}\left(\frac{4(n+t)}{n}, \frac{4(n+t)}{n}, \ldots, \frac{4(n+t)}{n}\right) .
$$

Hence, we find that $\mathcal{H}(\mathcal{Q})$ has the following eigenvalues

$$
\begin{gathered}
\lambda_{11}=0, \lambda_{22}=2\left(\frac{2 t}{n}+\frac{a(t)}{n-1}\right), \lambda_{33}=\cdots=\lambda_{n n}=2\left(\frac{n+t}{n}+\frac{a(t)}{n-1}\right), \\
\lambda_{i j}=4\left(\frac{n+t}{n}+\frac{a(t)}{n-1}\right), \lambda_{i n}=\frac{4(n+t)}{n},
\end{gathered}
$$

for all $i, j \in\{1,2, \ldots, n-1\}, i \neq j$. Thus, $\mathcal{Q}$ is parabolic and reaches at minimum $\mathcal{Q}\left(h^{c}\right)=0$ for the solution $h^{c}$ of the system (3.9). Hence $\mathcal{Q} \geq 0$ and hence we have

$$
2 \tau \leq t \mathcal{C}+a(t) \mathcal{C}(L)+n(n-1) f_{1}+6 f_{2}\left(d_{1} \cos ^{2} \theta_{1}+d_{2} \cos ^{2} \theta_{2}\right)
$$


whereby, we obtain

$$
\rho \leq \frac{t}{n(n-1)} \mathrm{C}+\frac{a(t)}{n(n-1)} \mathrm{C}(L)+f_{1}+\frac{6 f_{2}}{n(n-1)}\left(d_{1} \cos ^{2} \theta_{1}+d_{2} \cos ^{2} \theta_{2}\right),
$$

for every tangent hyperplane $L$ of $M$. If we take the infimum over all tangent hyperplane $L$, the result trivially follows. Moreover the equality sign holds if and only if

$$
h_{i j}^{\gamma}=0, \text { for all } i, j \in\{1, \ldots, n\}, i \neq j \text { and } \gamma \in\{n+1, \ldots, 2 m\}
$$

and

$$
h_{n n}^{\gamma}=\frac{n(n-1)}{t} h_{11}^{\gamma}=\cdots=\frac{n(n-1)}{t} h_{n-1 n-1}^{\gamma}, \text { for all } \gamma \in\{n+1, \ldots, 2 m\} .
$$

From (3.10) and (3.11), we obtain that the equality holds if and only if the submanifold is invariantly quasi-umbilical with normal connections in $\bar{M}$, such that the shape operator takes the form (3.3) with respect to the orthonormal tangent and orthonormal normal frames.

In the same way, we can prove (ii).

Theorem 3.1 yield the following result.

Corollary 3.1. Let $M$ be a bi-slant submanifold of a generalized complex space form $\bar{M}\left(f_{1}, f_{2}\right)$. Then

(i) the normalized $\delta$-Casorati curvature $\delta_{c}(n-1)$ satisfies

$$
\rho \leq \delta_{c}(n-1)+f_{1}+\frac{6 f_{2}}{n(n-1)}\left(d_{1} \cos ^{2} \theta_{1}+d_{2} \cos ^{2} \theta_{2}\right) .
$$

Moreover, the equality sign holds if and only if $M$ is an invariantly quasiumbilical submanifold with trivial normal connection in $\bar{M}\left(f_{1}, f_{2}\right)$, such that with respect to suitable tangent orthonormal frame $\left\{e_{1}, \ldots, e_{n}\right\}$ and normal orthonormal frame $\left\{e_{n+1}, \ldots, e_{2 m}\right\}$, the shape operator $S_{r} \equiv S_{e_{r}}, r \in\{n+1$, $\ldots, 2 m\}$, takes the following form

$$
S_{n+1}=\left(\begin{array}{cccccc}
a & 0 & 0 & \ldots & 0 & 0 \\
0 & a & 0 & \ldots & 0 & 0 \\
0 & 0 & a & \ldots & 0 & 0 \\
\vdots & \vdots & \vdots & \ddots & \vdots & \vdots \\
0 & 0 & 0 & \ldots & a & 0 \\
0 & 0 & 0 & \ldots & 0 & 2 a
\end{array}\right), S_{n+2}=\cdots=S_{2 m}=0, \text { and }
$$

(ii) the normalized $\delta$-Casorati curvature $\widehat{\delta}_{c}(n-1)$ satisfies

$$
\rho \leq \widehat{\delta}_{c}(n-1)+f_{1}+\frac{6 f_{2}}{n(n-1)}\left(d_{1} \cos ^{2} \theta_{1}+d_{2} \cos ^{2} \theta_{2}\right) .
$$

Moreover, the equality sign holds if and only if $M$ is an invariantly quasiumbilical submanifold with trivial normal connection in $\bar{M}\left(f_{1}, f_{2}\right)$, such that 
with respect to suitable tangent orthonormal frame $\left\{e_{1}, \ldots, e_{n}\right\}$ a and normal orthonormal frame $\left\{e_{n+1}, \ldots, e_{2 m}\right\}$, the shape operator $S_{r} \equiv S_{e_{r}}, r \in\{n+1$, $\ldots, 2 m\}$, takes the following form

$$
S_{n+1}=\left(\begin{array}{cccccc}
2 a & 0 & 0 & \ldots & 0 & 0 \\
0 & 2 a & 0 & \ldots & 0 & 0 \\
0 & 0 & 2 a & \ldots & 0 & 0 \\
\vdots & \vdots & \vdots & \ddots & \vdots & \vdots \\
0 & 0 & 0 & \ldots & 2 a & 0 \\
0 & 0 & 0 & \ldots & 0 & a
\end{array}\right), S_{n+2}=\ldots=S_{2 m}=0
$$

Proof. (i) One can easily see that

$$
\left[\delta_{c}\left(\frac{n(n-1)}{2}: n-1\right)\right]_{p}=n(n-1)\left[\delta_{c}(n-1)\right]_{p},
$$

at any point $p \in M$. Therefore, putting $t=\frac{n(n-1)}{2}$ in (3.1) and taking into account (3.12) we have our assertion.

Similarly,we obtain (ii).

Moreover, we have the following.

Theorem 3.2. Let $M$ be submanifolds of a generalized complex space form $\bar{M}\left(f_{1}, f_{2}\right)$. Then we have the following table for generalized normalized $\delta$-Casorati curvatures:

Table 2: Generalized Normalized $\delta$-Casorati curvatures

\begin{tabular}{|l|l|l|l|}
\hline$S . N$. & $\bar{M}\left(f_{1}, f_{2}\right)$ & $M$ & Inequality \\
\hline$(1)$ & $\bar{M}\left(f_{1}, f_{2}\right)$ & semi-slant & (a) $\rho \leq \frac{\delta_{c}(t ; n-1)}{n(n-1)}+f_{1}+\frac{6 f_{2}}{n(n-1)}\left(d_{1}+d_{2} \cos ^{2} \theta_{2}\right)$ \\
& & & (b) $\rho \leq \frac{\widehat{\delta_{c}(t ; n-1)}}{n(n-1)}+f_{1}+\frac{6 f_{2}}{n(n-1)}\left(d_{1}+d_{2} \cos ^{2} \theta_{2}\right)$ \\
& & & (a) $\rho \leq \frac{\delta_{c}(t ; n-1)}{n(n-1)}+f_{1}+\frac{6 f_{2}}{n(n-1)} d_{1} \cos ^{2} \theta_{1}$ \\
& & & (b) $\rho \leq \frac{\widehat{\delta_{c}(t ; n-1)}}{n(n-1)}+f_{1}+\frac{6 f_{2}}{n(n-1)} d_{1} \cos ^{2} \theta_{1}$ \\
& & & (a) $\rho \leq \frac{\delta_{c}(t ; n-1)}{n(n-1)}+f_{1}+\frac{6 f_{2}}{n(n-1)} d_{1}$ \\
& & & (b) $\rho \leq \frac{\widehat{\delta}_{c}(t ; n-1)}{n(n-1)}+f_{1}+\frac{6 f_{2}}{n(n-1)} d_{1}$ \\
& & & (3) \\
& & &
\end{tabular}




\begin{tabular}{|l|l|l|l|}
\hline$S . N$. & $\bar{M}\left(f_{1}, f_{2}\right)$ & $M$ & Inequality \\
\hline$(4)$ & $\bar{M}\left(f_{1}, f_{2}\right)$ & slant & (a) $\rho \leq \frac{\delta_{c}(t ; n-1)}{n(n-1)}+f_{1}+\frac{3 f_{2}}{(n-1)} \cos ^{2} \theta$ \\
& & & (b) $\rho \leq \frac{\widehat{\delta_{c}(t ; n-1)}}{n(n-1)}+f_{1}+\frac{3 f_{2}}{(n-1)} \cos ^{2} \theta$ \\
& & & (a) $\rho \leq \frac{\delta_{c}(t ; n-1)}{n(n-1)}+f_{1}+\frac{3 f_{2}}{(n-1)}$ \\
& & & (b) $\rho \leq \frac{\widehat{\delta_{c}(t ; n-1)}}{n(n-1)}+f_{1}+\frac{3 f_{2}}{(n-1)}$ \\
\hline$(6)$ & $\bar{M}\left(f_{1}, f_{2}\right)$ & invariant & (a) $\rho \leq \frac{\delta_{c}(t ; n-1)}{n(n-1)}+f_{1}$ \\
& & & (b) $\rho \leq \frac{\widehat{\delta_{c}(t ; n-1)}}{n(n-1)}+f_{1}$ \\
& & &
\end{tabular}

where in each case $0<t<n(n-1)$ and $t>n(n-1)$ for $(1)$ and $(2)$ respectively for any real number $t$. Moreover, the equality holds if and only if $M$ is an invariantly quasi-umbilical submanifold with trivial normal connection in $\bar{M}\left(f_{1}, f_{2}\right)$, such that with respect to suitable tangent orthonormal frame $\left\{e_{1}, \ldots, e_{n}\right\}$ and normal orthonormal frame $\left\{e_{n+1}, \ldots, e_{2 m}\right\}$, the shape operator $S_{r} \equiv S_{e_{r}}, r \in\{n+1, \ldots, 2 m\}$, takes the following form

$$
S_{n+1}=\left(\begin{array}{cccccc}
a & 0 & 0 & \ldots & 0 & 0 \\
0 & a & 0 & \ldots & 0 & 0 \\
0 & 0 & a & \ldots & 0 & 0 \\
\vdots & \vdots & \vdots & \ddots & \vdots & \vdots \\
0 & 0 & 0 & \ldots & a & 0 \\
0 & 0 & 0 & \ldots & 0 & \frac{n(n-1)}{t} a
\end{array}\right), S_{n+2}=\cdots=S_{2 m}=0 .
$$

Proof. First four results of the Theorem 3.2 can be simply obtained with the help of Table 1 and the results in Theorem 3.1. And the next two results of the Theorem 3.2 can be seen by putting $\theta=0$ and $\theta=\frac{\pi}{2}$ in case of invariant and anti-invariant submanifold respectively in the result of slant submanifold given in Theorem 3.2.

As the consequence of the last theorem, we note the following corollary.

Corollary 3.2. Let $M$ be submanifolds of a generalized complex space form $\bar{M}\left(f_{1}, f_{2}\right)$. Then for the normalized $\delta$-Casorati we have the following table 
Table 3: Normalized $\delta$-Casorati curvatures

\begin{tabular}{|c|c|c|c|}
\hline S.N. & $\bar{M}\left(f_{1}, f_{2}\right)$ & $M$ & Inequality \\
\hline$(1)$ & $\bar{M}\left(f_{1}, f_{2}\right)$ & semi-slant & $\begin{array}{l}\text { (a) } \rho \leq \delta_{c}(n-1)+f_{1}+\frac{6 f_{2}}{n(n-1)}\left(d_{1}+d_{2} \cos ^{2} \theta_{2}\right) \\
\text { (b) } \rho \leq \widehat{\delta}_{c}(n-1)+f_{1}+\frac{6 f_{2}}{n(n-1)}\left(d_{1}+d_{2} \cos ^{2} \theta_{2}\right)\end{array}$ \\
\hline$(2)$ & $\bar{M}\left(f_{1}, f_{2}\right)$ & hemi-slant & $\begin{array}{l}\text { (a) } \rho \leq \delta_{c}(n-1)+f_{1}+\frac{6 f_{2}}{n(n-1)} d_{1} \cos ^{2} \theta_{1} \\
\text { (b) } \rho \leq \widehat{\delta}_{c}(n-1)+f_{1}+\frac{6 f_{2}}{n(n-1)} d_{1} \cos ^{2} \theta_{1}\end{array}$ \\
\hline$(3)$ & $\bar{M}\left(f_{1}, f_{2}\right)$ & $C R$ & $\begin{array}{l}\text { (a) } \rho \leq \delta_{c}(n-1)+f_{1}+\frac{6 f_{2}}{n(n-1)} d_{1} \\
\text { (b) } \rho \leq \widehat{\delta}_{c}(n-1)+f_{1}+\frac{6 f_{2}}{n(n-1)} d_{1}\end{array}$ \\
\hline (4) & $\bar{M}\left(f_{1}, f_{2}\right)$ & slant & $\begin{array}{l}\text { (a) } \rho \leq \delta_{c}(n-1)+f_{1}+\frac{3 f_{2}}{(n-1)} \cos ^{2} \theta \\
\text { (b) } \rho \leq \widehat{\delta}_{c}(n-1)+f_{1}+\frac{3 f_{2}}{(n-1)} \cos ^{2} \theta\end{array}$ \\
\hline$(5)$ & $\bar{M}\left(f_{1}, f_{2}\right)$ & invariant & $\begin{array}{l}\text { (a) } \rho \leq \delta_{c}(n-1)+f_{1}+\frac{3 f_{2}}{(n-1)} \\
\text { (b) } \rho \leq \widehat{\delta}_{c}(n-1)+f_{1}+\frac{3 f_{2}}{(n-1)}\end{array}$ \\
\hline (6) & $\bar{M}\left(f_{1}, f_{2}\right)$ & anti-invariant & $\begin{array}{l}\text { (a) } \rho \leq \delta_{c}(n-1)+f_{1} \\
\text { (b) } \rho \leq \widehat{\delta}_{c}(n-1)+f_{1}\end{array}$ \\
\hline
\end{tabular}

Moreover, the equality sign for the inequalities $\delta_{c}$ in the above holds if and only if $M$ is an invariantly quasi-umbilical submanifold with trivial normal connection in $\bar{M}\left(f_{1}, f_{2}\right)$, such that with respect to suitable tangent orthonormal frame $\left\{e_{1}, \ldots, e_{n}\right\}$ and normal orthonormal frame $\left\{e_{n+1}, \ldots, e_{2 m}\right\}$, the shape operator $S_{r} \equiv S_{e_{r}}, r \in\{n+1, \ldots, 2 m\}$, takes the following form

$$
S_{n+1}=\left(\begin{array}{cccccc}
a & 0 & 0 & \ldots & 0 & 0 \\
0 & a & 0 & \ldots & 0 & 0 \\
0 & 0 & a & \ldots & 0 & 0 \\
\vdots & \vdots & \vdots & \ddots & \vdots & \vdots \\
0 & 0 & 0 & \ldots & a & 0 \\
0 & 0 & 0 & \ldots & 0 & 2 a
\end{array}\right), S_{n+2}=\cdots=S_{2 m}=0
$$


and the equality sign for the inequalities $\widehat{\delta}_{c}$ in the above table holds if and only if $M$ is an invariantly quasi-umbilical submanifold with trivial normal connection in $\bar{M}\left(f_{1}, f_{2}\right)$, such that with respect to suitable tangent orthonormal frame $\left\{e_{1}, \ldots, e_{n}\right\}$ and normal orthonormal frame $\left\{e_{n+1}, \ldots, e_{2 m}\right\}$, the shape operator $S_{r} \equiv S_{e_{r}}, r \in\{n+1, \ldots, 2 m\}$, takes the following form

$$
S_{n+1}=\left(\begin{array}{cccccc}
2 a & 0 & 0 & \ldots & 0 & 0 \\
0 & 2 a & 0 & \ldots & 0 & 0 \\
0 & 0 & 2 a & \ldots & 0 & 0 \\
\vdots & \vdots & \vdots & \ddots & \vdots & \vdots \\
0 & 0 & 0 & \ldots & 2 a & 0 \\
0 & 0 & 0 & \ldots & 0 & a
\end{array}\right), S_{n+2}=\ldots=S_{2 m}=0
$$

\section{Inequality for Doubly Warped Product Bi-Slant Submanifold}

Let $M_{1}$ and $M_{2}$ be Riemannian manifolds of dimension $n_{1}$ and $n_{2}$, equipped with Riemannian metric $g_{1}$ and $g_{2}$, respectively. Let $\sigma_{1}$ and $\sigma_{2}$ be positive differentiable functions on $M_{1}$ and $M_{2}$, respectively. Thus, the doubly warped product $M=$ ${ }_{\sigma_{2}} M_{1} \times{ }_{\sigma_{1}} M_{2}$ [17] of dimension $n$ is defined based on the product manifold $M_{1} \times M_{2}$ endowed with a metric $g=\sigma_{2}^{2} g_{1}+\sigma_{1}^{2} g_{2}$.

In this section, mainly we will prove the following.

Theorem 4.1. Let $\phi: M={ }_{\sigma_{2}} M_{1} \times_{\sigma_{1}} M_{2} \rightarrow \bar{M}\left(f_{1}, f_{2}\right)$ be an isometric immersion of an $n$-dimensional doubly warped product bi-slant submanifold of a $2 m$-dimensional generalized complex space form. Then

$$
\begin{aligned}
n_{2} \frac{\Delta_{1} \sigma_{1}}{\sigma_{1}}+n_{1} \frac{\Delta_{2} \sigma_{2}}{\sigma_{2}} \leq & \frac{n^{2}}{2}\|H\|^{2}+f_{1} n_{1} n_{2} \\
& +\frac{3}{2} f_{2}\left(n_{1} \cos ^{2} \theta_{1}+n_{2} \cos ^{2} \theta_{2}\right)-\frac{n}{2} \mathrm{C}+n \mathcal{C}(L),
\end{aligned}
$$

where $n_{i}=\operatorname{dim} M_{i}, i=1,2, n=n_{1}+n_{2}$ and $\Delta_{i}$ is Laplacian operator on $M_{i}, i=1,2$.

Proof. Let us assume that $\phi: M={ }_{\sigma_{2}} M_{1} \times{ }_{\sigma_{1}} M_{2} \rightarrow \bar{M}\left(f_{1}, f_{2}\right)$ be an isometric immersion of a warped product ${ }_{\sigma_{2}} M_{1} \times{ }_{\sigma_{1}} M_{2}$ into a generalized complex space form $\bar{M}\left(f_{1}, f_{2}\right)$. Suppose that $n_{1}, n_{2}$ and $n$ are the dimensions of $M_{1}, M_{2}$ and $M$, respectively. Then for the unit vector fields $X, Z$ tangent to $M_{1}, M_{2}$, respectively, it is easily seen that $[17]$

$$
K(X \wedge Z)=\frac{1}{\sigma_{1}}\left\{\left(\nabla_{X}^{1} X\right) \sigma_{1}-X^{2} \sigma_{1}\right\}+\frac{1}{\sigma_{2}}\left\{\left(\nabla_{Z}^{2} Z\right) \sigma_{2}-Z^{2} \sigma_{2}\right\} .
$$

Let us assume a local orthonormal frame $\left\{e_{1}, e_{2}, \ldots, e_{n}\right\}$ such that $e_{1}, e_{2}, \ldots, e_{n_{1}}$ are tangent to $M_{1}$ and $e_{n_{1}+1}, \ldots, e_{n}$ are tangent to $M_{2}$. Then

$$
n_{2} \frac{\Delta_{1} \sigma_{1}}{\sigma_{1}}+n_{1} \frac{\Delta_{2} \sigma_{2}}{\sigma_{2}}=\sum_{1 \leq i \leq n_{1}} \sum_{n_{1}+1 \leq j \leq n} K\left(e_{i} \wedge e_{j}\right) \text {. }
$$


Further, equations (2.4) and (4.2) imply

$$
n_{2} \frac{\Delta_{1} \sigma_{1}}{\sigma_{1}}+n_{1} \frac{\Delta_{2} \sigma_{2}}{\sigma_{2}}=\tau-\sum_{1 \leq j<k \leq n_{1}} K\left(e_{j} \wedge e_{k}\right)-\sum_{n_{1}+1 \leq s<t \leq n} K\left(e_{s} \wedge e_{t}\right) .
$$

Moreover, (2.1), (2.2) and (4.3) yield the following

$$
\begin{aligned}
n_{2} \frac{\Delta_{1} \sigma_{1}}{\sigma_{1}}+n_{1} \frac{\Delta_{2} \sigma_{2}}{\sigma_{2}}= & \tau-\frac{f_{1}}{2}\{n(n-1)\}+f_{1} n_{1} n_{2} \\
& -3 f_{2} \sum_{1 \leq j<k \leq n_{1}} g^{2}\left(e_{j}, J e_{k}\right)-3 f_{2} \sum_{n_{1}+1 \leq s<t \leq n} g^{2}\left(e_{s}, J e_{t}\right) \\
& -\sum_{r=n+1}^{2 m} \sum_{1 \leq j<k \leq n_{1}}\left(h_{j j}^{r} h_{k k}^{r}-\left(h_{j k}^{r}\right)^{2}\right) \\
& -\sum_{r=n+1}^{2 m} \sum_{n_{1}+1 \leq s<t \leq n}\left(h_{s s}^{r} h_{t t}^{r}-\left(h_{s t}^{r}\right)^{2}\right) .
\end{aligned}
$$

Using (2.5), (2.6) and (4.4), we obtain

$$
\begin{aligned}
n_{2} \frac{\Delta_{1} \sigma_{1}}{\sigma_{1}}+n_{1} \frac{\Delta_{2} \sigma_{2}}{\sigma_{2}}= & -\frac{f_{1}}{2}\{n(n-1)\}+f_{1} n_{1} n_{2} \\
& -\frac{3}{2} f_{2}\left(n_{1} \cos ^{2} \theta_{1}+n_{2} \cos ^{2} \theta_{2}\right)-\frac{1}{2} n_{1}^{2}\left\|H_{1}\right\|^{2} \\
& -\frac{1}{2} n_{2}^{2}\left\|H_{2}\right\|^{2}+\frac{1}{2} n_{1} \mathrm{C}(L)+\frac{1}{2} n_{2} \mathrm{C}(L) \\
& \leq \tau-\frac{f_{1}}{2}\{n(n-1)\}+f_{1} n_{1} n_{2} \\
& \left.-\frac{3}{2} f_{2}\left(n_{1} \cos ^{2} \theta_{1}+n_{2} \cos ^{2} \theta_{2}\right)+\frac{1}{2} n \mathcal{C}(L)\right),
\end{aligned}
$$

where $H_{1}$ and $H_{2}$ are partial mean curvature vectors of $M_{1}$ and $M_{2}$ respectively.

An immediate consequence of the Theorem 4.1 is the following obstruction result.

Corollary 4.1. Let $\phi: M={ }_{\sigma_{2}} M_{1} \times{ }_{\sigma_{1}} M_{2} \rightarrow \bar{M}\left(f_{1}, f_{2}\right)$ be an isometric immersion of an n-dimensional doubly warped product bi-slant submanifold $M$ in $2 m$-dimensional generalized complex space form $\bar{M}\left(f_{1}, f_{2}\right)$ such that $\sigma_{1}$ and $\sigma_{2}$ are harmonic functions. Then immersion of $M$ can not be flat in $\bar{M}\left(f_{1}, f_{2}\right)$ if

$$
f_{1} \leq \frac{1}{2 n_{1} n_{2}-n^{2}+n}\left[f_{2}\left(n_{1} \cos ^{2} \theta_{1}+n_{2} \cos ^{2} \theta_{2}\right)-n \mathcal{C}(L)\right] .
$$

Next, we state and prove.

Theorem 4.2. Let $\phi: M={ }_{\sigma_{2}} M_{1} \times{ }_{\sigma_{1}} M_{2} \rightarrow \bar{M}\left(f_{1}, f_{2}\right)$ be an isometric immersion of an $n$-dimensional doubly warped product submanifolds of a $2 m$-dimensional generalized complex space form. Then we have following table: 
Table 4: Doubly warped product inequalities

\begin{tabular}{|c|c|c|c|}
\hline$S . N$. & $\bar{M}\left(f_{1}, f_{2}\right)$ & $M$ & Inequality \\
\hline (1) & $\bar{M}\left(f_{1}, f_{2}\right)$ & semi-slant & $\begin{array}{l}n_{2} \frac{\Delta_{1} \sigma_{1}}{\sigma_{1}}+n_{1} \frac{\Delta_{2} \sigma_{2}}{\sigma_{2}} \leq \tau-\frac{f_{1}}{2}\{n(n-1)\}+f_{1} n_{1} n_{2}- \\
\left.\frac{3}{2} f_{2}\left(n_{1}+n_{2} \cos ^{2} \theta_{2}\right)+\frac{1}{2} n \mathcal{C}(L)\right)\end{array}$ \\
\hline (2) & $\bar{M}\left(f_{1}, f_{2}\right)$ & hemi-slant & $\begin{array}{l}n_{2} \frac{\Delta_{1} \sigma_{1}}{\sigma_{1}}+n_{1} \frac{\Delta_{2} \sigma_{2}}{\sigma_{2}} \leq \tau-\frac{f_{1}}{2}\{n(n-1)\}+f_{1} n_{1} n_{2}- \\
\left.\frac{3}{2} f_{2}\left(n_{1} \cos ^{2} \theta_{1}\right)+\frac{1}{2} n \mathcal{C}(L)\right)\end{array}$ \\
\hline (3) & $\bar{M}\left(f_{1}, f_{2}\right)$ & $C R$ & $\begin{array}{l}n_{2} \frac{\Delta_{1} \sigma_{1}}{\sigma_{1}}+n_{1} \frac{\Delta_{2} \sigma_{2}}{\sigma_{2}} \leq \tau-\frac{f_{1}}{2}\{n(n-1)\}+f_{1} n_{1} n_{2}- \\
\left.\frac{3}{2} f_{2} n_{1}+\frac{1}{2} n \mathcal{C}(L)\right)\end{array}$ \\
\hline (4) & $\bar{M}\left(f_{1}, f_{2}\right)$ & slant & $\begin{array}{l}n_{2} \frac{\Delta_{1} \sigma_{1}}{\sigma_{1}}+n_{1} \frac{\Delta_{2} \sigma_{2}}{\sigma_{2}} \leq \tau-\frac{f_{1}}{2}\{n(n-1)\}+f_{1} n_{1} n_{2}- \\
\left.\frac{3}{2} f_{2} n \cos ^{2} \theta+\frac{1}{2} n \mathcal{C}(L)\right)\end{array}$ \\
\hline (5) & $\bar{M}\left(f_{1}, f_{2}\right)$ & invariant & $\begin{array}{l}n_{2} \frac{\Delta_{1} \sigma_{1}}{\sigma_{1}}+n_{1} \frac{\Delta_{2} \sigma_{2}}{\sigma_{2}} \leq \tau-\frac{f_{1}}{2}\{n(n-1)\}+f_{1} n_{1} n_{2}- \\
\left.\frac{3}{2} f_{2} n+\frac{1}{2} n \mathcal{C}(L)\right)\end{array}$ \\
\hline (6) & $\bar{M}\left(f_{1}, f_{2}\right)$ & anti-invariant & $\begin{array}{l}n_{2} \frac{\Delta_{1} \sigma_{1}}{\sigma_{1}}+n_{1} \frac{\Delta_{2} \sigma_{2}}{\sigma_{2}} \leq \tau-\frac{f_{1}}{2}\{n(n-1)\}+f_{1} n_{1} n_{2}+ \\
\left.\frac{1}{2} n \mathrm{C}(L)\right)\end{array}$ \\
\hline
\end{tabular}

Proof. We obtain the first four results of the Theorem 4.2 directly by using Table 1 and the result of the Theorem 4.1 and last two results by putting $\theta=0$ and $\theta=\frac{\pi}{2}$ respectively in result of slant submanifold.

\section{REFERENCES}

[1] M. Aquib, Bounds for generalized normalized $\delta$-Casorati curvatures for bi-slant submanifolds in T-space forms, Filomat (to appear).

[2] D. Blair and A. Ledger, Quasi-umbilical minimal submanifolds of Euclidean space, Bull. Belg. Math. Soc. Simon Stevin 51 (1977), 3-22.

[3] F. Casorati, Mesure de la courbure des surface suivant 1'idee commune. Ses rapports avec les mesures de coubure gaussienne et moyenne, Acta Math. 14 (1999), 95-110.

[4] B.Y. Chen, Relationship between Ricci curvature and shape operator for submanifolds with arbitrary codimensions, Glasg. Math. J. 41 (1999), 33-41.

[5] B.Y. Chen, Some pinching and classification theorems for minimal submanifolds, Arch. Math. 60 (1993), 568-578.

[6] S. Decu, S. Haesen and L. Verstralelen, Optimal inequalities involving Casorati curvatures, Bull. Transilv. Univ. Brasov Ser. B 49 (2007), 85-93.

[7] V. Ghisoiu, Inequalities for the Casorati curvatures of the slant submanifolds in complex space forms, Riemannian geometry and applications. Proceedings RIGA 2011, ed. Univ. Bucuresti, Bucharest (2011), 145-150.

[8] A. Gray, Nearly Kaehler manifolds, J. Differential Geom. 4 (1970), 283-309.

[9] S. Haesen, D. Kowalczyk and L. Verstralelen, On the extrinsic principal directions of Riemannnian submanifolds, Note Mat. 29 (2009), 41-53.

[10] S. Hong, K. Matsumoto and M.M. Triphati, Certian basic inequalities for submanifolds of locally conformal kaehler space forms, SUT J. Math. 41(1) (2005), 75-94. 
[11] T. Kashiwada, Some properties of locally conformal Kaehler manifolds, Hokkaido Math. J. 8 (1979), 191-198.

[12] D. Kowalczyk, Casorati curvatures, Bull. Transilv. Univ. Brasov Ser. III 50(1) (2008), 2009-2013.

[13] J.S. Kim, Y.M. Song and M.M. Tripathi, B. Y. Chen inequalities for submanifolds in generilized complex space forms, Bull. Korean Math. Soc. 40(3) (2003), 411-423.

[14] C.W. Lee, J.W. Lee, G.E. Vilcu and D.W. Yoon, Optimal inequalities for the Casorati curvatures of the submanifolds of Generalized space form endowed with seni-symmetric metric connections, Bull. Korean Math. Soc. 52 (2015), 1631-1647.

[15] K. Matsumoto, I. Mihai and A. Oiaga, Ricci curvature of submanifolds in complex space form, Rev. Roumaine Math. Pures Appl. 46 (2001), 775-782.

[16] F. Tricerri and L. Vanhecke, Curvature tensors on almost Hermitian manifolds, Trans. Amer. Math. Soc. 26 (2003), 85-94.

[17] B. Unal, Doubly warped products, Thesis(Ph.D.), University of Missouri, Columbia, 2000.

[18] L. Vanhecke, Almost Hermitian manifolds with J-invariant Riemann curvature tensor, Rend. Semin. Mat. Univ. Politec. Torino 34 (1975-76), 487-498.

[19] L. Verstralelen, Geometry of submanifolds I, The first Casorati curvature indicatrices, Kragujevac J. Math. 37 (2013), 5-23.

${ }^{1}$ Department of Mathematics,

FACUlty of NATURAL SCIENCES,

Jamia Millia ISLAMia,

New DeLHI-110025, IndiA

Email address: aquib80@gmail.com

Email address: hasan_jmi@yahoo.com

${ }^{2}$ Department of Mathematics,

Al-FAlah UNIVERSity,

HARYANA-121004, INDIA

Email address: jamali_dbd@yahoo.co.in 